\title{
CONSTANT-ENVELOPE PRECODING FOR SATELLITE SYSTEMS
}

\author{
Christos G. Tsinos, Aakash Arora and Björn Ottersten \\ SnT-Interdisciplinary Centre for Security, Reliability and Trust, Univ. of Luxembourg, Luxembourg \\ e-mails: \{christos.tsinos,aakash.arora,bjorn.ottersten\}@uni.lu
}

\begin{abstract}
In this paper, Constant-Envelope Precoding techniques are presented for satellite-based communication systems. In the developed transmission technique the signals of the antennas are designed to be of constant amplitude, improving the robustness of the latter to the nonlinear distortions on satellite systems, introduced by the employed on-board Traveling-Wave-Tube-Amplifiers. We consider the forward link of a multi-beam broadband satellite system where the aim is to design the signals at the gateway such that the desired symbols are transmitted to the intended user terminals and the transmitted signals from the satellite terminal are of constant amplitude. At first, the gateway signals are designed given that a fixed on-board beamformer is applied to the satellite terminal. Then, the case of an adaptive onboard beamformer is considered which is designed jointly with the gateway signals. The design of the gateway signals and the adaptive on-board beamformer, in the second case, requires solving difficult nonconvex problems. Efficient algorithmic solutions are developed based on the saddle point method. The effectiveness of the proposed approaches is verified via numerical results.
\end{abstract}

Index Terms - Constant Envelope Precoding, On-board Beamforming, Ground-based Precoding, Non-linear Precoding, Satellite Communications

\section{INTRODUCTION}

Communications satellites based on the "bent-pipe" technology [1] can be viewed as non-regenerative relays that simply filter and amplify the received signals from the Gateway Terminal (GT). Even though they exhibit these limited processing capabilities, they are able to handle simultaneously many streams of information targeted to different User Terminals (UTs). The multi-antenna Satellite Terminal (ST) is usually equipped with an On-Board BeamForming (OBBF) network which may be tuned in order to optimize the transmission towards specific UTs. As the on-line adaptation of the OBBF is sometimes a demanding task, an On-Ground BeamForming (OGBF) technology was applied in some recent multi-beam satellite systems [2]-[3]. In OGBF, the signals to be transmitted by the ST are optimized at the GT and thus, this eliminates the need for a complex on-board beamformer. On the other hand, the OGBF technology requires the exchange of all the feed signals between the ST and the GT resulting in increased bandwidth demands on the feeder link side.

Usually, the GT-to-ST communication is established through channels orthogonal in frequency and thus, interference is not a problem in those links. In contrast, the ST-to-UTs links experience strong interference due to the full-frequency reuse communication

This work was supported by FNR, Luxembourg under the project CORE ECLECTIC regime and the possible side-lobes of the different radiating elements of the ST. To that end, several works have tried to address the interference mitigation problem by extending the concept of linear precoding in satellite terminals. By the term linear precoding, we refer to the precoders that depend only on the Channel State Information (CSI) and thus, can be updated according to the channel coherence time. Their simplicity led to the development of numerous different solutions tailored for the various system models that have appeared in the wireless communications literature over the past years [4]-[6]. In the context of satellite systems, several linear precoding-based solutions have been proposed for different communication regimes over the past years [3][7]-[11]. These approaches aim at the design of the OGBF signals and are usually combined with fixed or adaptive OBBF networks.

In the literature of wireless communications, an alternative approach to design the transmitter's signals is based on the so-called "Constant-Envelope Precoding (CEP)" techniques [12]-[17]. The latter are nonlinear or symbol level precoders [18] that are based on both the CSI and the transmitted information symbols to the UTs. In CEP, the transmitted signals are designed to be of constant modulus regardless of the CSI and the information symbols that are conveyed to the UTs. Having signals of constant modulus across the transmitter's antennas in satellite systems results in more robust designs to nonlinear distortions. The latter are due to the employment of Traveling-Wave-Tube-Amplifiers (TWTAs) on-board the satellite systems that introduce distortion to each one of the emitted signals by the antennas that depends on instantaneous transmitted power [19]. Thus, having high variations of the instantaneous transmission power per antenna, determines different phase shifts in the amplification stages, resulting in further performance degradation [19]-[21].

Despite their profound advantages, CEP techniques tailored for satellite communication systems have yet to be developed. This motivated us to study this topic in the present paper. Analytically, the contributions of the present paper are as follows. A multi-beam satellite system is considered based on a multi-antenna ST that serves multiple UTs and a single GT. For this system, CEP techniques are developed for both the cases of a fixed and an adaptive OBBF network. In the first case, the OGBF signals are optimized such that the transmitted signals from the ST are of constant envelope. In the second case, the OBBF network's coefficients are jointly optimized with the OGBF signals, as well. Both of the approaches require the solution to difficult nonconvex problems that have yet to be addressed in the literature. To that end, efficient solutions for them are developed via the Saddle Point (SP) framework [22]. The effectiveness of the proposed solutions is verified via simulations and compared to the one of typical linear precoding approaches based on the Minimum Mean Square Error (MMSE) criterion.

The rest of the paper is organized as follows. Sec. 2 describes the considered system model and defines the optimization problems to be addressed. Sec. 3 derives the algorithmic solution to the de- 
fined optimization problems. Sec. 4 presents the numerical results and Sec. 5 concludes this work.

\section{SYSTEM MODEL}

Let us consider the forward link of a broadband satellite system with a single GT that aims at the data transmission to a large number UTs. Under the assumption of a transparent satellite payload, a Time Division Multiple Access approach is followed according to which the GT serves simultaneously a total of $M$ UTs per symbol time.

The ST is assumed to be equipped with an array fed reflector antenna of $N \geq M$ number of feeds /elements. Moreover, the UTs are based on single antenna receivers. The GT-to-ST and the ST-to-UTs links are established through different frequency areas. Thus, they are implemented via a Full Duplex mode that is assumed to preserve the orthogonality between the corresponding transmissions. On the other hand, all the links from the ST to the $M$ UTs are established via the same frequency spectrum and hence, they are corrupted by interference.

Now, by dropping the time index for simplicity, the received signals at the UTs on a symbol time are given by,

$$
\mathbf{y}=\mathbf{H} \mathbf{x}_{f}+\mathbf{z},
$$

where $\mathbf{y}$ is the $M \times 1$ vector of the received signals at the UTs, $\mathbf{x}_{f}$ is the $N \times 1$ on-board transmit signal vector at the feed space, respectively, the $M \times N$ matrix $\mathbf{H}$ contains the coefficients of the channels between the GT and the served UTs and are modeled as in [3] and the $M \times 1$ vector $\mathbf{n}$ contains the Additive White Gaussian Noise (AWGN) that corrupts the corresponding transmissions.

In the following two different approaches for designing a transmit signal that possesses the constant-envelope property in the feed space are developed. The proposed solutions are developed with the view to transmit a desired symbol $\mathbf{s}(m) \in \mathcal{O}, 1 \leq m \leq M$ from the GT to the $m$ th UT such that a performance metric is optimized. $\mathcal{O}$ is the set of the employed constellation points.

\subsection{On-board Fixed Beamforming \& Nonlinear Precoding}

The first case involves a fixed, pre-determined and channel independent $N \times K$ beamforming matrix $\mathbf{B}$ applied on-board such that

$$
\mathbf{x}_{f}=\mathbf{B} \tilde{\mathbf{x}}_{b}
$$

where $\tilde{\mathbf{x}}_{b}$ is the $K \times 1$ ground transmitted signal in the beam space under the assumption of a perfectly calibrated and noiseless feeder link. In this case, $\tilde{\mathbf{x}}_{b}$ is designed via a nonlinear precoding approach such that the transmission is optimized subject to the constant-envelope constraints on the overall signal $\mathbf{x}_{f}$ in (2).

By assuming that in the timeslot under consideration, the GT aims at the transmission of a symbol $s(m), 1 \leq m \leq M$ at the $m$ th UT, we may design the signal $\tilde{\mathbf{x}}_{b}$ as the solution to the following optimization problem, given by

$$
\begin{gathered}
\left(\mathcal{P}_{1}\right): \quad \min _{\mathbf{x}_{b}, q} \frac{1}{2}\left\|\mathbf{s}-q \mathbf{H B} \mathbf{x}_{b}\right\|_{2}^{2}, \\
\text { s.t. } \mathbf{B x}_{b} \in \Omega^{N \times 1} \\
|q|^{2} \leq P_{\max }
\end{gathered}
$$

where $\mathbf{s}$ is the $M \times 1$ vector that has the stack of symbols that are desired to be transmitted to each one of the UTs, i.e., $\mathbf{s}=$ $[s(1), \ldots, s(M)]^{T},(\cdot)^{T}$ is the transpose of a vector, $\Omega$ is the set of unit-modulus complex numbers, that is $\Omega=\{\omega \in \mathbb{C}|| \omega \mid=1\}$ and $P_{\max }$ is a transmit power constraint at the feed space. Note that without loss of the generality, we have assumed that $\tilde{\mathbf{x}}_{b}=q \mathbf{x}_{b}$, where $q \in \mathbb{C}$ is a scalar complex number. The modulus of $q$ can be seen as the common modulus of all the entries in $\mathbf{B} \tilde{\mathbf{x}}_{b}$ while its argument may be applied as a phase shift in the arguments of the aforementioned vector. With this approach, the level of the constant-envelope can be optimized, as well, as long as it respects the limitations posed by the PA which are introduced to the problem through the constraint $|q|^{2} \leq P_{\max }$.

Problem $\left(\mathcal{P}_{1}\right)$ is nonconvex and in general NP-hard [23]. In Sec. 3.1 , an efficient solution based on the SP framework is developed.

\subsection{On-board Adaptive Beamforming \& Nonlinear Precoding}

In the second approach, it is assumed that the on-board beamformer may be adapted in order to follow the channel and symbol variations such that improved performance is achieved. It is assumed that the OBBF matrix is fixed for a block of $L$ symbols. By stacking the received signals at the $M$ UTs, the transmit signals at the beam space and the noise variable in matrices, $\mathbf{Y}=\left[\mathbf{y}^{(1)}, \ldots, \mathbf{y}^{(L)}\right]$, $\tilde{\mathbf{X}}_{b}=\left[\tilde{\mathbf{x}}_{b}^{(1)}, \ldots, \tilde{\mathbf{x}}_{b}^{(L)}\right]$ and $\mathbf{Z}=\left[\mathbf{z}^{(1)}, \ldots, \mathbf{z}^{(L)}\right]$, respectively, we may express the input-output relationship of the system for the block of $L$ symbols in matrix form by

$$
\mathbf{Y}=\mathbf{H B} \tilde{\mathbf{X}}_{b}+\mathbf{Z}
$$

Let us now stack the symbol vectors to be transmitted to the UTs during these $L$ symbol times in a matrix $\mathbf{S}=\left[\mathbf{s}^{(1)}, \ldots, \mathbf{s}^{(L)}\right]$. Then, the matrix of the transmission signals of these symbol times, $\tilde{\mathbf{X}}_{b}$, is derived by solving the following optimization problem defined as

$$
\begin{aligned}
&\left(\mathcal{P}_{2}\right): \quad \min _{\mathbf{x}_{b}, \mathbf{Q}, \mathbf{B}} \frac{1}{2}\left\|\mathbf{S}-\mathbf{H B X}_{b} \mathbf{Q}\right\|_{F}^{2}, \\
& \text { s.t. } \quad \mathbf{B X} \mathbf{X}_{b}(l) \in \Omega^{N \times 1}, \quad 1 \leq l \leq L \\
& \quad|\mathbf{Q}(l, l)|^{2} \leq P_{\max }, \quad 1 \leq l \leq L
\end{aligned}
$$

where $\mathbf{Q}$ is a diagonal matrix with non-zero entries $\mathbf{Q}(l, l) \in \mathbb{C}$ that can be seen as the common modulus of $\mathbf{B} \tilde{\mathbf{X}}_{b}(l)$ with $\tilde{\mathbf{X}}_{b}(l)$ denoting the $l$ th column of matrix $\tilde{\mathbf{X}}_{b}$, in a similar manner to the on-board fixed beamforming case in Sec. 2.1.

Problem $\left(\mathcal{P}_{2}\right)$ is also nonconvex and NP-hard [23]. It is in general, more difficult than the corresponding one for the fixed beamforming case $\left(\mathcal{P}_{2}\right)$. This is the case since, the optimization problem involves $L$ times the variables to be optimized as compared to $\left(\mathcal{P}_{1}\right)$ as the transmitted signals for a block of $L$ symbols are simultaneously optimized. On top of that, matrix $\mathbf{B}$ is an additional matrix variable that has to be optimized via the problem. In the Sec. 3.2, an efficient solution also based on the SP framework is developed.

\section{SOLUTION}

In this section, the solutions to $\left(\mathcal{P}_{1}\right)$ and $\left(\mathcal{P}_{2}\right)$ are derived in two separate subsections that follow.

\subsection{On-board Fixed Beamforming and Nonlinear Precoding}

Let us start from the optimization problem $\left(\mathcal{P}_{1}\right)$. Since the OBBF matrix $\mathbf{B}$ is fixed, the variables to be optimized are the signal at the feed space $\mathbf{x}_{b}$ and its envelope level $q$. At first, the $N \times 1$ auxiliary 
variable $\mathbf{u}$ is introduced into the problem in order to express it in the equivalent form, given by

$$
\begin{aligned}
\left(\mathcal{P}_{3}\right): \quad \min _{\mathbf{u}, \mathbf{x}_{b}, q} & \frac{1}{2}\|\mathbf{s}-q \mathbf{u}\|_{2}^{2}, \\
\text { s.t. } \mathbf{u} & \in \Omega^{N \times 1} \\
\mathbf{u} & =\mathbf{B} \mathbf{x}_{b} \\
|q|^{2} & \leq P_{\max } .
\end{aligned}
$$

The partial AuGmented Lagrangian function (AGL) of $\left(\mathcal{P}_{3}\right)$ with respect the constraint $\mathbf{u}=\mathbf{B} \mathbf{x}_{b}$ is given by,

$\mathcal{L}\left(\mathbf{u}, \mathbf{x}_{b}, q, \boldsymbol{\lambda}\right)=\frac{1}{2}\|\mathbf{s}-q \mathbf{u}\|_{2}^{2}+\boldsymbol{\lambda}^{H}\left(\mathbf{u}-\mathbf{B} \mathbf{x}_{b}\right)+\frac{\alpha}{2}\left\|\mathbf{u}-\mathbf{B} \mathbf{x}_{b}\right\|_{2}^{2}$,

where $\boldsymbol{\lambda} \in \mathbf{C}^{N \times 1}$ is the Lagrange multiplier vector, $(\cdot)^{H}$ is the hermitian of a complex vector/matrix and $\alpha$ is a scalar positive real penalty parameter.

Now based on (4), the solution to $\left(\mathcal{P}_{3}\right)$ may be approximated by solving in its place, the one defined by

$$
\left(\mathcal{P}_{4}\right): \quad \min _{\mathbf{u} \in \Omega^{N \times 1}, \mathbf{x}_{b} \in \mathbb{C}^{N \times 1}} \max _{\boldsymbol{\lambda} \in \mathbb{C}^{2} \leq P_{\max } \times 1} \mathcal{L}\left(\mathbf{u}, \mathbf{x}_{b}, q, \boldsymbol{\lambda}\right) .
$$

Problem $\left(\mathcal{P}_{4}\right)$ is the dual problem of $\left(\mathcal{P}_{3}\right)$ derived through the Lagrangian relaxation of the equality constraints of the latter. Furthermore, it is in a form that can be addressed by developing an algorithm based on a variation of the Arrow-Hurwicz SP method [22][24]. This method is based on the fact that the optimal values for the primaldual variables are saddle points of the Lagrangian function and employs first order methods to find the solution. It is a simple and efficient way to tackle constrained optimization problems with established convergence results when applied to convex problems. Little is known regarding its performance when applied to nonconvex problems. Nevertheless, some recent literature results showed that first order methods may be applied successfully to solve nonconvex problems [25] and thus, since such methods are the core to ArrowHurwicz SP method, we were motivated to seek solutions towards that direction.

Moving along with the derivation of the solution, the SP method employs gradient descent updates for the primal variables and gradient ascent ones for the dual variables at each iteration of the algorithm. Thus, for $\left(\mathcal{P}_{4}\right)$ the updates are given by

$$
\begin{aligned}
\mathbf{u}_{n} & =\Pi_{\Omega}\left\{\mathbf{u}_{n-1}-\mu_{n} \nabla_{\mathbf{u}} \mathcal{L}\left(\mathbf{u}_{n-1}, \mathbf{x}_{b, n-1}, q_{n-1}, \boldsymbol{\lambda}_{n-1}\right)\right\} \\
\mathbf{x}_{b, n} & =\mathbf{x}_{b, n-1}-\mu_{n} \nabla_{\mathbf{x}_{b}} \mathcal{L}\left(\mathbf{u}_{n-1}, \mathbf{x}_{b, n-1}, q_{n-1}, \boldsymbol{\lambda}_{n-1}\right) \\
q_{n} & =\Pi_{\mathcal{Q}}\left\{q_{n-1}-\mu_{n} \nabla_{q} \mathcal{L}\left(\mathbf{u}_{n-1}, \mathbf{x}_{b, n-1}, q_{n-1}, \boldsymbol{\lambda}_{n-1}\right)\right\} \\
\boldsymbol{\lambda}_{n} & =\boldsymbol{\lambda}_{n-1}+\mu_{n} \nabla_{\boldsymbol{\lambda}} \mathcal{L}\left(\mathbf{u}_{n-1}, \mathbf{x}_{b, n-1}, q_{n-1}, \boldsymbol{\lambda}_{n-1}\right)
\end{aligned}
$$

where $\mu_{n}$ is a step-size parameter, $\nabla_{x}$ denotes the gradient with respect the variable $x, \Pi_{\Omega}\{\cdot\}$ is the element-wise projection operator of a vector onto the set $\Omega$, defined in Sec. 2.1 and $\Pi_{\mathcal{Q}}\{\cdot\}$ is the projection of a complex number over the set $\mathcal{Q}$, defined as $\mathcal{Q}=\left\{q \in \mathbb{C} \|\left. q\right|^{2} \leq P_{\max }\right\}$.

For the step-size parameter a nonsummable diminishing step length rule is selected, i.e. $\mu_{n}=\gamma_{n} /\left\|\mathbf{g}_{n}\right\|_{2}^{2}$, where $\gamma_{n} \geq 0$, $\lim _{n \rightarrow \infty} \gamma_{n}=0, \sum_{n=1}^{\infty} \gamma_{n}=\infty$ and

$$
\mathbf{g}_{n}=\left[\begin{array}{c}
\nabla_{\mathbf{u}} \mathcal{L}\left(\mathbf{u}_{n-1}, \mathbf{x}_{b, n-1}, q_{n-1}, \boldsymbol{\lambda}_{n-1}\right) \\
\nabla_{\mathbf{x}_{b}} \mathcal{L}\left(\mathbf{u}_{n-1}, \mathbf{x}_{b, n-1}, q_{n-1}, \boldsymbol{\lambda}_{n-1}\right) \\
\nabla_{q} \mathcal{L}\left(\mathbf{u}_{n-1}, \mathbf{x}_{b, n-1}, q_{n-1}, \boldsymbol{\lambda}_{n-1}\right) \\
\nabla_{\boldsymbol{\lambda}} \mathcal{L}\left(\mathbf{u}_{n-1}, \mathbf{x}_{b, n-1}, q_{n-1}, \boldsymbol{\lambda}_{n-1}\right)
\end{array}\right] .
$$

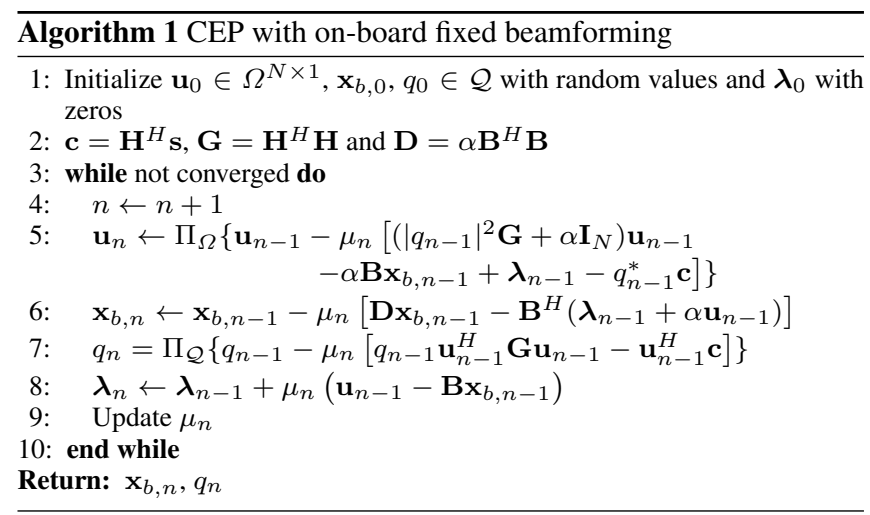

The projection operator $\Pi_{\Omega}\{\cdot\}$ is calculated as follows. According to the definition of $\left(\mathcal{P}_{4}\right), \mathbf{u}$ must lie in $\Omega^{N \times 1}$. This can be enforced by solving the following optimization problem

$$
\begin{aligned}
\left(\mathcal{P}_{5}\right): & \min _{\mathbf{u}_{\Omega}}\left\|\mathbf{u}_{\Omega}-\mathbf{u}\right\|_{2}^{2} \\
& \text { s.t. } \quad \mathbf{u}_{\Omega} \in \Omega^{N \times 1},
\end{aligned}
$$

where $\mathbf{u}_{\Omega}$ is the projection of $\mathbf{u}$ onto the set $\Omega^{N \times 1}$. Clearly, $\left(\mathcal{P}_{5}\right)$ is separable in each one of the elements, in vectors $\mathbf{u}$ and $\mathbf{u}_{\Omega}$ and admits the closed form given by

$$
\mathbf{u}_{\Omega}(k)=\left\{\begin{array}{ll}
1, & \mathbf{u}(k)=0 \\
\frac{\mathbf{u}(k)}{|\mathbf{u}(k)|}, & \mathbf{u}(k) \neq 0
\end{array} .\right.
$$

In a similar manner, it can be shown that the projection onto the set $\mathcal{Q}$ admits the closed form given by,

$$
\Pi_{\mathcal{Q}}\{q\}=\left\{\begin{array}{cl}
q, & |q|^{2} \leq P_{\max } \\
\sqrt{P_{\max } \frac{q}{|q|},} & |q|^{2}>P_{\max }
\end{array} .\right.
$$

The algorithm updates in an iterative manner the variables $\mathbf{u}_{n}, \mathbf{x}_{b, n}$, $q_{n}$ and $\boldsymbol{\lambda}_{n}$ until a convergence criterion is met. The complete procedure is shown in Algorithm 1 for completeness. The variables shown in line 2 of the algorithm are pre-calculated to reduce the required computational complexity. For the convergence of Algorithm 1, we present Theorem 1 bellow. The proof may be found in [26].

Theorem 1: Let $\left\{\mathbf{u}_{n}, \mathbf{x}_{b, n}, q_{n}, \boldsymbol{\lambda}_{n}\right\}$ is a sequence generated by the steps in Algorithm 1. Let us further assume that $\mu_{n}=\gamma_{n} /\left\|\mathbf{g}_{n}\right\|_{2}^{2}$, where $\gamma_{n} \geq 0, \lim _{n \rightarrow \infty} \gamma_{n}=0, \sum_{n=1}^{\infty} \gamma_{n}=\infty$ and $\sum_{n=1}^{\infty} \gamma_{n}^{2}=\theta \leq \infty$. Then, the sequence $\left\{\mathbf{u}_{n}, \mathbf{x}_{b, n}, q_{n}, \boldsymbol{\lambda}_{n}\right\}$ converges to a limit point $\left\{\mathbf{u}_{\star}, \mathbf{x}_{b, \star}, q_{\star}, \boldsymbol{\lambda}_{\star}\right\}$ that satisfies the KKT conditions of $\left(\mathcal{P}_{3}\right)$ and by equivalence the ones of $\left(\mathcal{P}_{1}\right)$, as well.

\subsection{On-board Adaptive Beamforming \& Nonlinear Precoding}

We may now move to the derivation of the solution to optimization problem $\left(\mathcal{P}_{2}\right)$. Now, the OBBF matrix is also optimized and thus, the entries of an additional $N \times K$ matrix variable have to be calculated by the proposed algorithmic solution. On top of that, the gateway signals may be optimized now on a block of symbols basis, as it can be seen from the definition of $\left(\mathcal{P}_{2}\right)$. Nevertheless, the same methodology with the fixed OBBF case may be employed once more. To that end, $\left(\mathcal{P}_{2}\right)$ is first transformed to an equivalent form by the use of the auxiliary $N \times L$ matrix variable $\mathbf{U}$, given by 


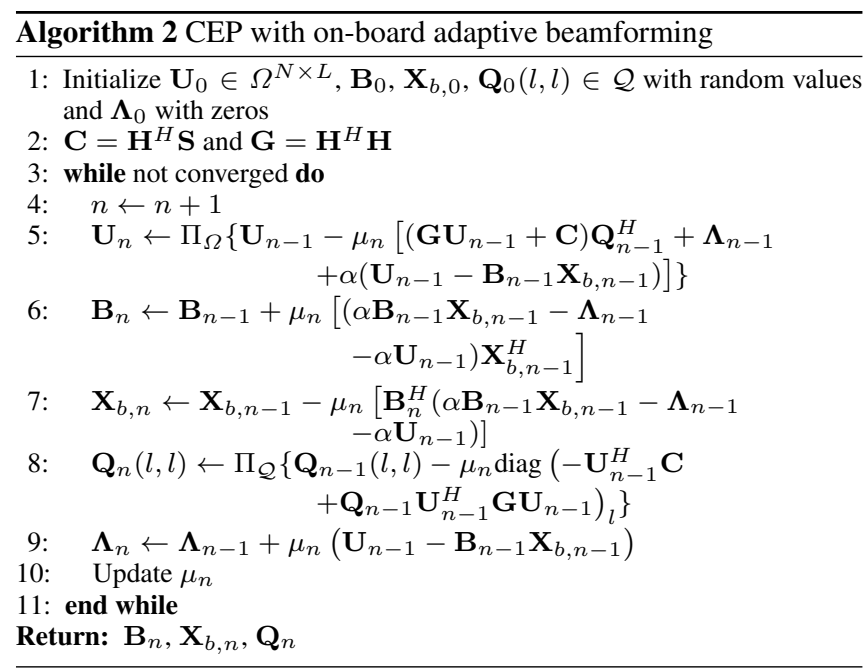

$$
\begin{aligned}
\left(\mathcal{P}_{6}\right): \quad \min _{\mathbf{x}_{b}, \mathbf{Q}, \mathbf{B}} \frac{1}{2}\|\mathbf{S}-\mathbf{H U Q}\|_{F}^{2}, \\
\text { s.t. } \mathbf{U} \in \Omega^{N \times L} \\
\quad \mathbf{U}=\mathbf{B} \mathbf{X}_{b} \\
\quad|\mathbf{Q}(l, l)|^{2} \leq P_{\max }, \quad 1 \leq l \leq L .
\end{aligned}
$$

Now, the partial AGL function of $\left(\mathcal{P}_{6}\right)$ with respect the constraint $\mathbf{U}=\mathbf{B X}_{b}$ is given by,

$$
\begin{aligned}
\mathcal{L}^{\prime}\left(\mathbf{U}, \mathbf{B}, \mathbf{X}_{b}, \mathbf{Q}, \boldsymbol{\Lambda}\right)= & \frac{1}{2}\|\mathbf{S}-\mathbf{U} \mathbf{Q}\|_{F}^{2}+\left\langle\boldsymbol{\Lambda}, \mathbf{U}-\mathbf{B X}_{b}\right\rangle \\
& +\frac{\alpha}{2}\left\|\mathbf{U}-\mathbf{B X}_{b}\right\|_{F}^{2},
\end{aligned}
$$

where $\left\langle\mathbf{A}_{1}, \mathbf{A}_{2}\right\rangle=\sum_{k, l} \mathbf{A}_{1}^{*}(k, l) \mathbf{A}_{2}(k, l)$, for two matrices $\mathbf{A}_{1}$ and $\mathbf{A}_{2}, \boldsymbol{\Lambda} \in \mathbb{C}^{N \times L}$ is the Lagrange Multiplier matrix and $\alpha$ is again a scalar penalty parameter.

We now once again use the Lagrangian relaxation concept on problem $\left(\mathcal{P}_{6}\right)$ which is approximated by its dual given by,

$\left(\mathcal{P}_{7}\right):$

$$
\min _{\substack{\mathbf{U} \in \Omega^{N \times L}, \mathbf{B} \in \mathbb{C}^{N \times N} \\ \mathbf{x}_{b} \in \mathbb{C}^{N \times L},|\mathbf{Q}(l, l)|^{2} \leq P_{\max }}} \max _{\boldsymbol{\Lambda} \in \mathbb{C}^{N \times L}} \mathcal{L}^{\prime}\left(\mathbf{U}, \mathbf{B}, \mathbf{X}_{b}, \mathbf{Q}, \boldsymbol{\Lambda}\right) .
$$

A solution for $\left(\mathcal{P}_{7}\right)$ is proposed based again on a variation of the Arrow-Hurwicz SP method, as it is done for the fixed OBBF case in Sec. 3.1. The derivation of the solution follows the same steps with the ones presented in Sec. 3.1 and thus, it is omitted in order to avoid unnecessary repetition. The complete procedure for solving $\left(\mathcal{P}_{7}\right)$ is given in Algorithm 2. The matrices $\mathbf{C}$ and $\mathbf{G}$ (line 2) are pre-calculated for computational complexity savings. Furthermore, the $\operatorname{diag}(\cdot)$ operator that appears in line 8 of the algorithm extracts the diagonal elements of a matrix into a vector form and $(\cdot)_{l}$ denotes its $l$ th entry. The projection operators $\Pi_{\Omega}$ (line 5 ) and $\Pi_{\mathcal{Q}}$ (line 8 ) can be found in (9) and (10), respectively. The step-size parameter is again set as in the case of the fixed OBBF case. Before closing this section, it is instructive to mention that similar convergence guarantees to the ones appearing in Theorem 1 can be found in [26] for Algorithm 2.

\section{NUMERICAL RESULTS AND CONCLUSIONS}

In this section, numerical results are shown for evaluating the performance of the techniques. An ST of $N=30$ antennas is assumed

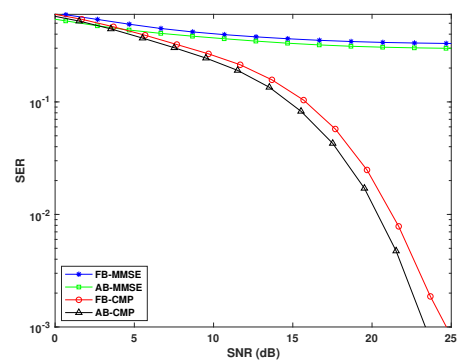

(a)

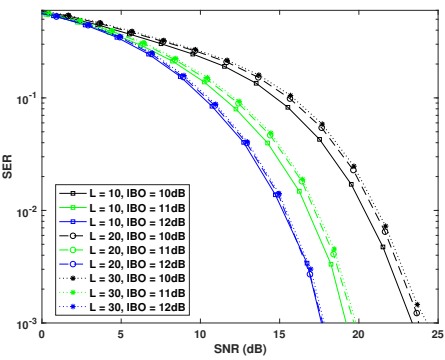

(b)
Fig. 1. SER curves of the different schemes for a ST of $N=30$ antennas that serves $M=10$ UTs. a) Comparison with linear MMSE-based precoders for IBO $=10 \mathrm{~dB}$ and b) Impact of the symbol block size on the performance of the adaptive OBBF-based CEP technique for differnt IBO values.

that serves $M=10$ UTs with $K=30$. The symbols to be transmitted to the UTs are drawn uniformly from an 8 - Phase Shift Keying (PSK) modulation. The step-size parameters are set such that the optimal performance is attained in the corresponding case. In order to model the effects of the TWTAs on the signal to be transmitted, the memory less Saleh TWT model was employed [27]. The selected model is tuned based on the "Input BackOff (IBO)" value defined as IBO $=10 \log _{10}\left(P_{0}^{I N} / P^{I N}\right)$ where $P_{0}^{I N}$ is the input power corresponding to the maximum output power (saturation power) of the TWTAs and $P^{I N}$ is the mean signal power signal at the input of the amplifiers. The performance is examined with respect the average uncoded Symbol Error Rate (SER) achieved by each one of the techniques for different transmit Signal-to-Noise-Ratio (SNR) values. The transmit SNR here is defined as $\mathbb{E}\left\{\left\|\mathbf{x}_{f}(l)\right\|_{2}^{2}\right\} / \sigma_{z}^{2}$. The results are averaged over 1000 channel realizations.

In Fig.1a, the performance of the proposed CEP technique based on the fixed OBBF network - Algorithm 1 ("FB-CEP") and of the one based on the adaptive OBBF network - Algorithm 2 ("AB-CEP") are depicted for $\mathrm{IBO}=10 \mathrm{~dB}$. For the one based on the adaptive OBBF network, the symbol block size is set to $L=10$. Furthermore, in the same figure, the performance of linear precoding solutions based on the MMSE criterion [3][26] are also depicted for both the fixed and adaptive OBBF cases. As it can be seen, the performance of the CEP based approaches are significantly better than the corresponding MMSE-based ones that present floor error even for low SNR values. This verifies the robustness of the former to the phase and amplitude corruptions introduced by the TWTAs due to the spatial constant-envelope property of the transmitted signals. On the contrary, the required overhead is much higher than that of a linear precoder since now the precoder must be adapted on a symbol time basis. Moreover, the performance of the precoding solution for the adaptive OBBF network is superior to the one for the fixed OBBF network, as in the former case there are more degrees of freedom that can be optimized to transmit the symbols to the UTs.

In Fig 1.b, the impact of the block symbol length $L$ on the performance of Algorithm 2 is examined for $L=\{10,20,30\}$ for different IBO values. As, it is shown, the increase on the $L$ results on a slight degradation on the performance since the adaptive OBBF matrix remains static for a larger block of symbols. On the contrary, for larger $L$, the overhead for the adaptation of the OBBF matrix is smaller. Furthermore, this gap on the performance is observed to decrease with an increase in the IBO value. This is the case, since for higher block lengths, Algorithm 2 tends to converge in solutions that result in higher transmit power for achieving a specific SER value compared to the corresponding ones for smaller $L$ values. Thus, the phase and noise corruptions due to the TWTAs are more severe for higher $L$ values which leads to the observed degradation when the IBO value is decreasing, as well. 


\section{REFERENCES}

[1] D. Minoli, Innovations in satellite communication and satellite technology, Wiley Online Library, 2015.

[2] J Tronc, P Angeletti, Nuan Song, Martin Haardt, J Arendt, and Gennaro Gallinaro, "Overview and comparison of on-ground and on-board beamforming techniques in mobile satellite service applications," International J. Satellite Commun. Netw., vol. 32, no. 4, pp. 291-308, 2014.

[3] B. Devillers, A. Perez-Neira, and C. Mosquera, "Joint linear precoding and beamforming for the forward link of multi-beam broadband satellite systems," in IEEE Global Telecommun. Conf. (GLOBECOM), Dec 2011, pp. 1-6.

[4] M. Bengtsson and B. Ottersten, "Optimal and suboptimal transmit beamforming," 2001.

[5] M. Schubert and H. Boche, "Solution of the multiuser downlink beamforming problem with individual SINR constraints," IEEE Trans. on Veh. Technol., vol. 53, no. 1, pp. 18-28, Jan 2004.

[6] Q. H. Spencer, A. L. Swindlehurst, and M. Haardt, "Zeroforcing methods for downlink spatial multiplexing in multiuser MIMO channels," IEEE Trans. Signal Process., vol. 52, no. 2, pp. 461-471, Feb 2004.

[7] G. Zheng, S. Chatzinotas, and B. Ottersten, "Generic optimization of linear precoding in multibeam satellite systems," IEEE Trans. Wireless Commun., vol. 11, no. 6, pp. 2308-2320, June 2012.

[8] D. Christopoulos, S. Chatzinotas, and B. Ottersten, "Multicast multigroup precoding and user scheduling for frame-based satellite communications," IEEE Trans. Wireless Commun., vol. 14, no. 9, pp. 4695-4707, Sep. 2015.

[9] V. Joroughi, M. . Vzquez, and A. I. Prez-Neira, "Precoding in multigateway multibeam satellite systems," IEEE Trans. Wireless Commun., vol. 15, no. 7, pp. 4944-4956, July 2016.

[10] V. Joroughi, M. . Vzquez, and A. I. Prez-Neira, "Precoding in multigateway multibeam satellite systems," IEEE Trans. Wireless Commun., vol. 15, no. 7, pp. 4944-4956, July 2016.

[11] C. Mosquera, R. Lpez-Valcarce, T. Ramrez, and V. Joroughi, "Distributed precoding systems in multi-gateway multibeam satellites: Regularization and coarse beamforming," IEEE Trans. Wireless Commun., vol. 17, no. 10, pp. 6389-6403, Oct 2018.

[12] C. Molln, E. G. Larsson, and T. Eriksson, "Waveforms for the massive MIMO downlink: Amplifier efficiency, distortion, and performance," IEEE Trans. Commun., vol. 64, no. 12, pp. 5050-5063, Dec 2016.

[13] S. K. Mohammed and E. G. Larsson, "Constant-envelope multi-user precoding for frequency-selective massive MIMO systems," IEEE Wireless Commun. Lett., vol. 2, no. 5, pp. 547550, October 2013.

[14] S. K. Mohammed and E. G. Larsson, "Single-user beamforming in large-scale MISO systems with per-antenna constantenvelope constraints: The doughnut channel," IEEE Trans.
Wireless Commun., vol. 11, no. 11, pp. 3992-4005, November 2012.

[15] S. K. Mohammed and E. G. Larsson, "Per-antenna constant envelope precoding for large multi-user MIMO systems," IEEE Trans. Commun., vol. 61, no. 3, pp. 1059-1071, March 2013.

[16] F. Liu, C. Masouros, P. V. Amadori, and H. Sun, "An efficient manifold algorithm for constructive interference based constant envelope precoding," IEEE Signal Process. Lett., vol. 24, no. 10, pp. 1542-1546, Oct 2017.

[17] S. Domouchtsidis, C. G. Tsinos, S. Chatzinotas, and B. Ottersten, "Symbol-level precoding for low complexity transmitter architectures in large-scale antenna array systems," IEEE Trans. Wireless Commun., vol. 18, no. 2, pp. 852-863, Feb 2019.

[18] M. Alodeh, D. Spano, A. Kalantari, C. Tsinos, D. Christopoulos, S. Chatzinotas, and B. Ottersten, "Symbol-level and multicast precoding for multiuser multiantenna downlink: A stateof-the-art, classification and challenges," IEEE Commun. Surveys Tuts., pp. 1-1, 2018.

[19] D. Spano, M. Alodeh, S. Chatzinotas, J. Krause, and B. Ottersten, "Spatial papr reduction in symbol-level precoding for the multi-beam satellite downlink," in 18th IEEE International Workshop on Signal Process. Adv. in Wireless Commun. (SPAWC), July 2017, pp. 1-5.

[20] D. Spano, M. Alodeh, S. Chatzinotas, and B. Ottersten, "Symbol-level precoding for the nonlinear multiuser miso downlink channel," IEEE Trans. Signal Process., vol. 66, no. 5, pp. 1331-1345, March 2018.

[21] Danilo Spano, Symeon Chatzinotas, Stefano Andrenacci, Jens Krause, and Björn Ottersten, "Per-antenna power minimization in symbol-level precoding for the multibeam satellite downlink," Int. J. Satellite Commun. Netw., vol. 37, no. 1, pp. 15-30, 2019.

[22] A. Nedić and A. Ozdaglar, "Subgradient methods for saddlepoint problems," J. optimization theory and applications, vol. 142, no. 1, pp. 205-228, 2009.

[23] S. Zhang and Y. Huang, "Complex quadratic optimization and semidefinite programming," SIAM J. Optimization, vol. 16, no. 3, pp. 871-890, 2006.

[24] K. J. Arrow, L. Hurwicz, and H. Uzawa, Studies in linear and non-linear programming, Cambridge Univ. Press, 1958.

[25] J. Tranter, N. D. Sidiropoulos, X. Fu, and A. Swami, "Fast unit-modulus least squares with applications in beamforming," IEEE Trans. Signal Process., vol. 65, no. 11, pp. 2875-2887, June 2017.

[26] C. G. Tsinos, A. Arora, and B. Ottersten, "Constant-modulus nonlinear precoding techniques for satellite-based communication systems," Under Preparation.

[27] A. A. M. Saleh, "Frequency-independent and frequencydependent nonlinear models of twt amplifiers," IEEE Trans. Commun., vol. 29, no. 11, pp. 1715-1720, November 1981. 